\title{
Role of embedded miniscrews in the placement of vestibular included canines using the lingual technique
}

\section{F. Haïm}

\section{ODF Qualified Specialist}

\author{
SUMMARY
}

The maxillary canine comes after the third molar as the most frequently included tooth. The prevalence of maxillary canine impact is $0.8 \%-2.8 \%$. Buccally included canines represent a minority of cases, approximately $15 \%-32 \%$ of the cases depending on the authors. The placement in the arch is often more complex due to periodontal considerations, the frequent proximity of the lateral incisor root and the very precise control of the direction of the tension to be delivered by the device. In this clinical case, the combination of two innovative techniques - submerged temporary anchorage device and customized lingual device-made it possible to decrease both the complexity of the treatment for the practitioner and the constraints for the patient.

\section{KEYWORDS}

Buccal included canine, miniscrew anchorage, customized lingual device

\section{INTRODUCTION}

Orthodontists are frequently confronted with the late discovery of included canines. The prevalence of maxillary included canines is $0.8 \%-2.8 \% \%^{3,78}$. According to previous studies ${ }^{5-7}$, those in a vestibular position represent $15 \%-32 \%$ of cases. Obtaining the position of the upper vestibular canines is a challenge for orthodontists who must decide between the risk of altering the lateral incisor root, which is linked to too much vertical traction and the periodontal risk linked to an excessive vestibular traction. The orthodontic device must be extremely precise in the application of the forces and directional variations of traction ${ }^{5}$ during the movement.

\section{Address for correspondence:}

Frédéric Haïm

11 rue de la Convention - 75015 Paris

E-mail: haim.frederic@wanadoo.fr 
Colombe, an 18-year-old female, consulted us after the discovery of a left temporary maxillary canine which is impacted on the arcade.

The clinical examination did not detect any other abnormality, except a very moderate coronovestibular version of 22 and the absence of germs for 18,38 , and 48 .

Additional investigations showed that 23 was impacted in a high position. The cuspidal tip of 23 was located between the apices of 21 and 22, in contact with the vestibular cortex while its root is above 22, 24, and 25 and its apex was in contact with the palatal cortex. The canine was therefore palatal at its most distal point, but its cuspidal tip, which is vestibular compared to 22, prohibited placement by palatal traction. A careful clinical examination made it possible to deduce the vestibular situation of 23 even before performing complementary examinations. (The coronovestibular version of 22 signified a radiculopalatal version, a consequence of the vestibular situation of the cuspidal tip of 23). Moreover, 63, had a resorption greater than three-fourths of its root and the space necessary for the establishment of 23 is insufficient.

Colombe was apprehensive regarding the installation of a visible orthodontic device, which seems to constitute for her an insurmountable obstacle. She said "I do not want braces at 18 years of age."

\section{Therapeutic goals and treatment plan}

A treatment plan was made. The goal was to set up 23 with an invisible and very simple device for the first part of the treatment, i.e., the

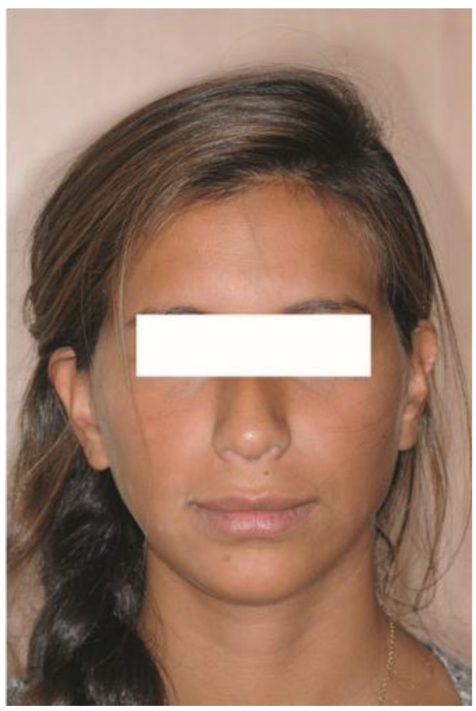

a

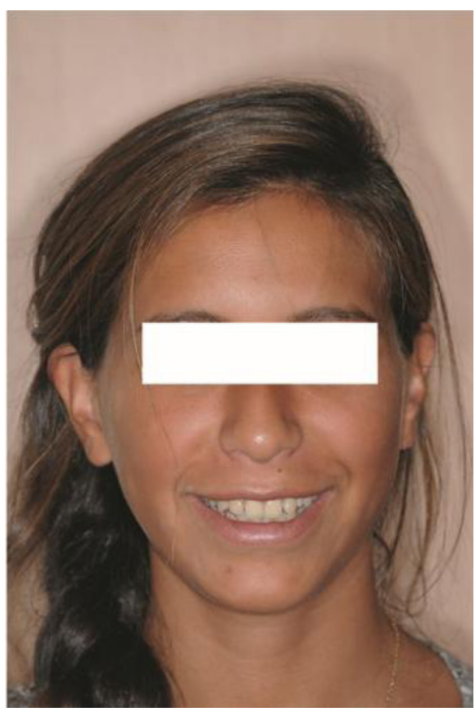

b

Figure 1

a through c: Start of treatment.

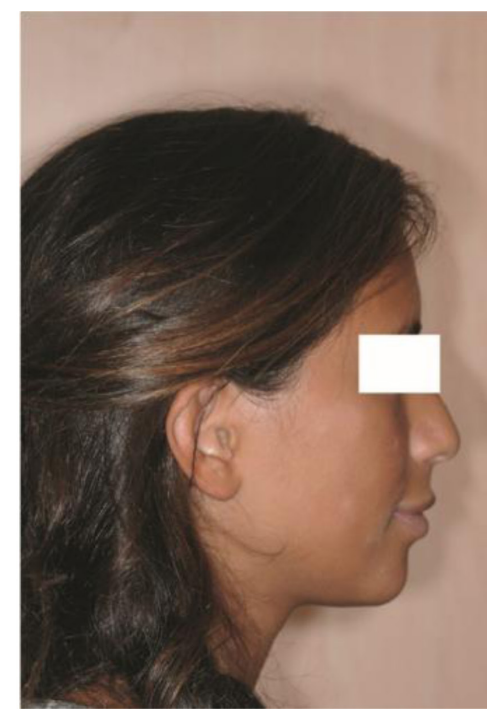

C 


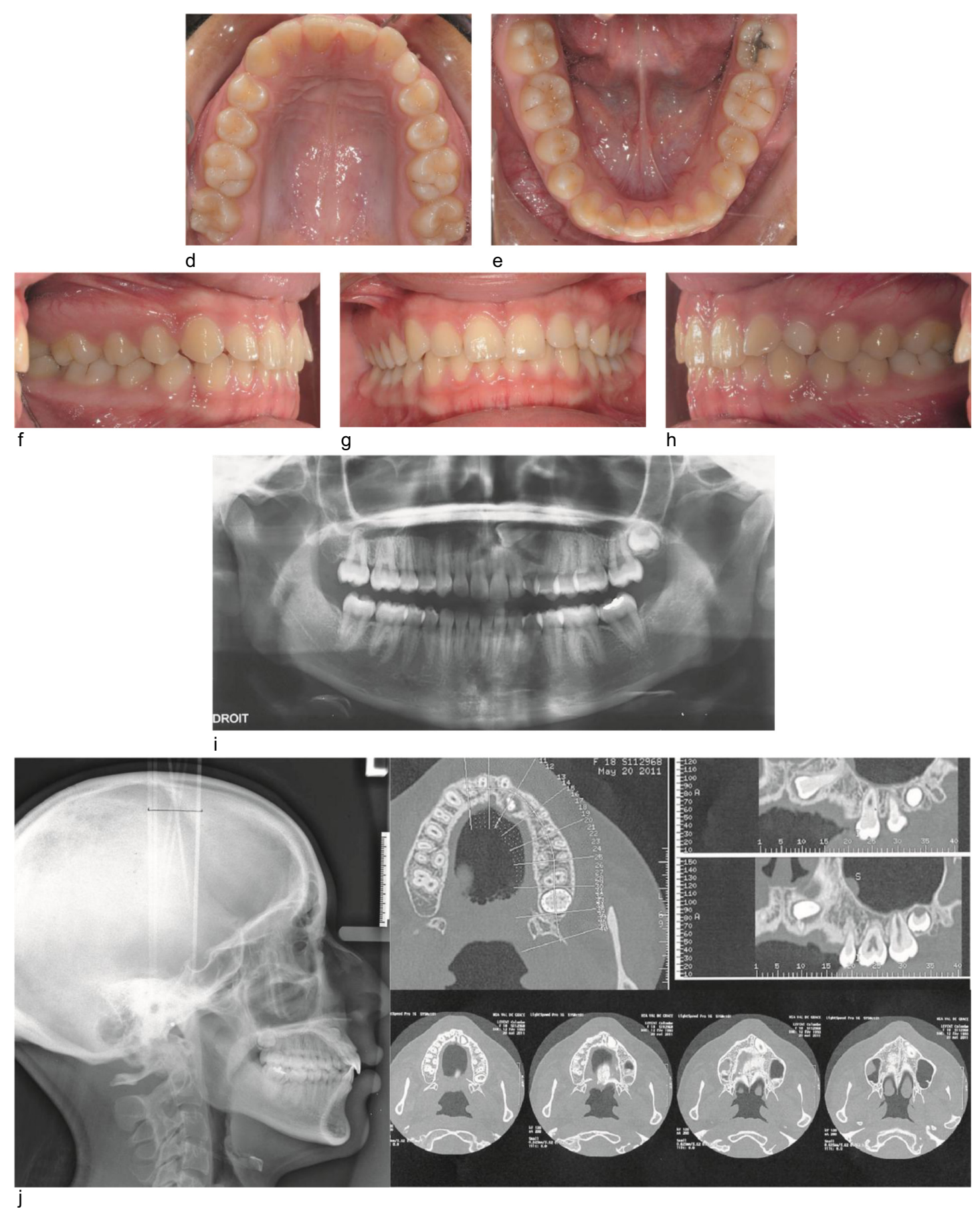

Figure 1

$d$ through j: Start of treatment. 
long submucosal route to the spontaneous canine eruption site. After that, 23 will be taken care of by an invisible orthodontic device for its aesthetic and functional positioning, after the avulsion of 63. Colombe provided her consent for this treatment proposal.

\section{Treatment plan}

The submucosal path of 23 would be created by a CT8 device described by $D$ and J-G Chillès with two miniscrews embedded ${ }^{2}$ (Tekka $3 \mathrm{~mm}$ ) on a TMA connection of 0.018 " with two spiral loops (Fig. 2). The removal of the crown of 23 and the bonding of the anchoring would be performed during the same

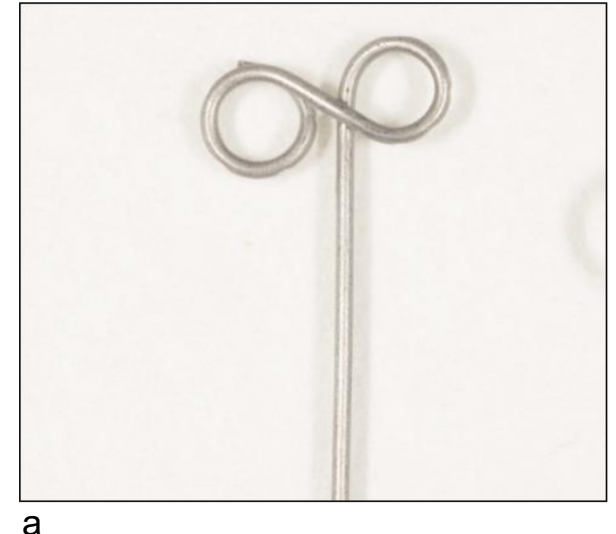

a

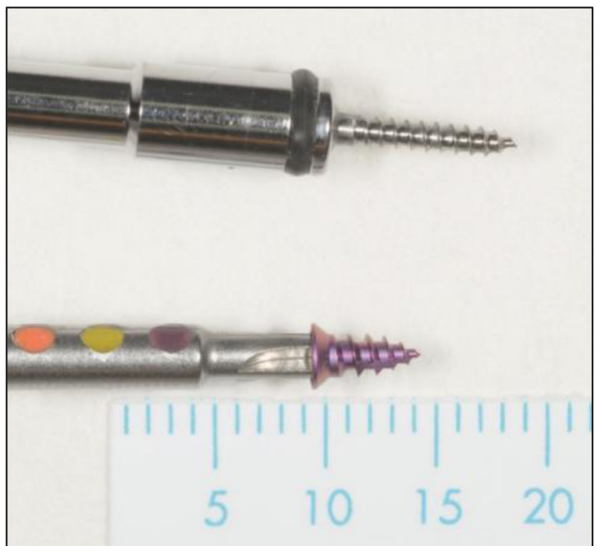

C

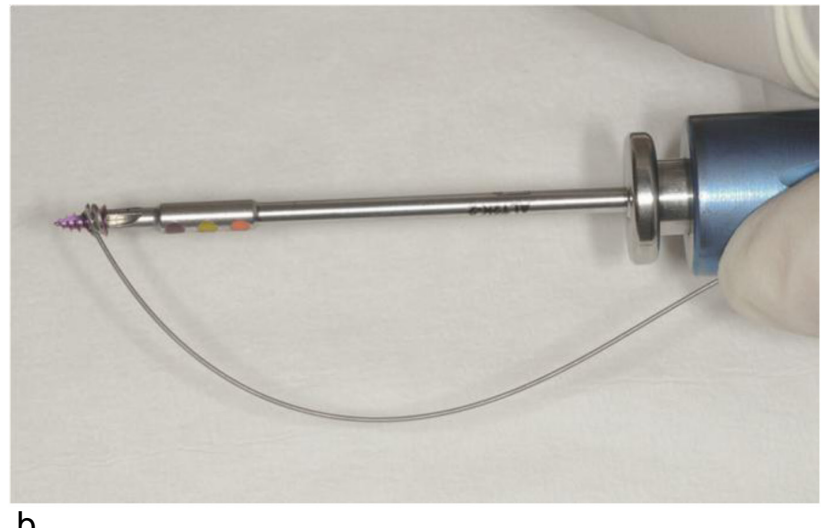

b

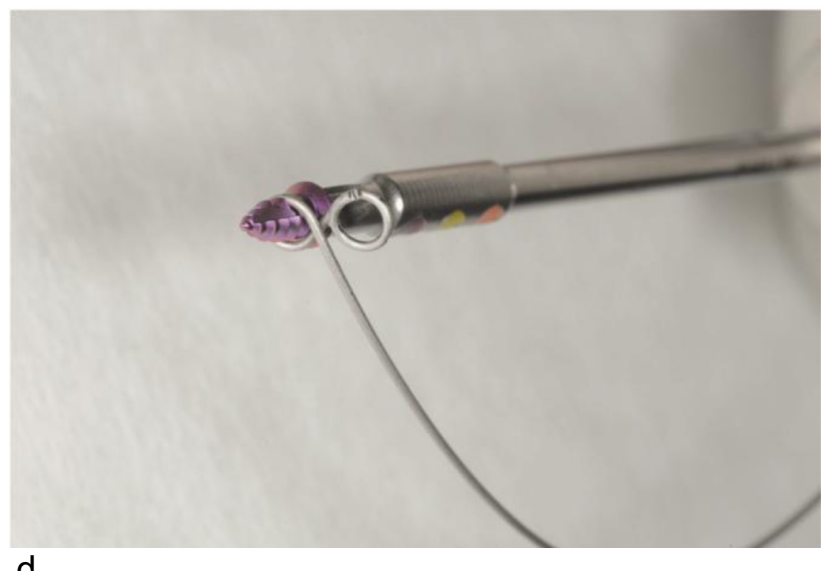

d

Figure 2

(a) A double screw is shaped with a Tweed clamp (middle activator) for the embedded end of the connector on a TMA 0.018" wire. Its diameter must be wide enough to allow the passage of the intracortical part of the screw and hold back the head. The connector is preformed and sterilized. (b) The 3-cm long Tekka embedded miniscrew is compared with an 8- $\mathrm{mm}$ dual-top transmucosal miniscrew that will be used at the end of treatment for the correction of a canine class II, induced by a undesirable effect during alignment. $c$ and d) Holding the screwdriver and

connectors for its implementation. A second Tekka screw will be placed in the other coil. 
procedure. The creation of the necessary space and the final placement of 23 would be made by an individual lingual device called "Win" as described by Dirk Wiechman ${ }^{9}$.

\section{Summary and discussion}

The choice of miniscrews and CT8 connections by $D$ and J-G Chillès was guided by the desire to obtain a weak but constant force directed toward the vestibule to avoid the root of 22 move toward the plane of occlusion and distally ${ }^{2}$. Any cantilever system anchored on the vestibular surface of the teeth is necessarily limited in its activation by the short length of the "active arm" . Embedding the screws limits the risk of bacterial percolation ${ }^{1-2}$ and the system is completely discrete. The lingual device "Win" was chosen for its small size and how easy it is to control the anterior torque on the canine slope during the insertion of a vertical "Edgewise ribbon" arch in vertical grooves very close to the palatal surface of the teeth ${ }^{9}$.

Only the maxillary arch was paired to decrease discomfort. This choice is not recommended because a loss of anchorage, induced by the alignment, was responsible for a vestibular version of the incisors and the appearance of a Class-II canine. Without the possibility of using Class-II elastics, this side effect necessitated a mass regression of the maxillary arch with the help of transmucosal miniscrews placed at the end of treatment.

\section{Treatment steps}

Step 1: Vestibular canine removal and bonding of the anchorage. The removal was directed by Jean-Marc Dersot. The strand emerges at the base of the vestibule opposite the most sloping point of the collar of 22. Then the screws and the CT8 round TMA 0.018" wire connectors are installed. An arciform incision at the end of the vestibule under the zygomatic process of the maxilla is performed. A full-thickness flap is lifted clearing the bone surface. The first screw, inserted in the distal loop of the connector, is placed blindly followed by the second screw. The loops are shaped so as to block the mesial arm of the connector. The natural deactivation of the loop accentuates the occlusodistal movement of the active arm (the system is not symmetrical to the right or to the left). A thumb-spring shapes the TMA 0.018" into a bayonet shape to remove it from the bone and pull it into the vestibule. The connector is cut, a terminal turn is shaped, and it is inserted into the loop of the wire strand (Figure 3).

Step 2: Only two modifications to the connections, made in the mouth, were necessary during this long treatment stage to provide a more occlusal and distal traction on the canine (Figs. 3 and 4).

Step 3: When the canine has reached the point of emergence, the multi-attachment device is placed and 22 is leveled and aligned (Fig. 5), the avulsion of 63 is set up and a cosmetic cover is bonded on, leaving a hole for the passage of an elastomeric "hollow tube" wire which is easy to tie. This wire is knotted on the $0.016 " \times 0.024$ " steel arch and will create a perfectly vertical traction through the cosmetic element.

Step 4: A second extraction is performed ${ }^{4}$ and shows the crown of the canine with a rotation of $120^{\circ}$. An 


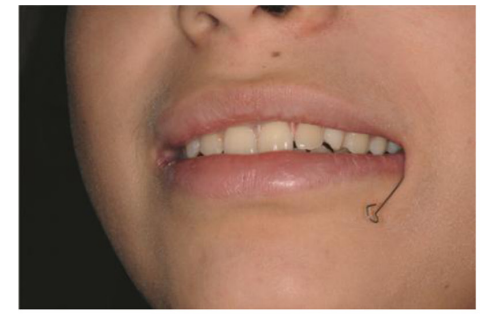

a

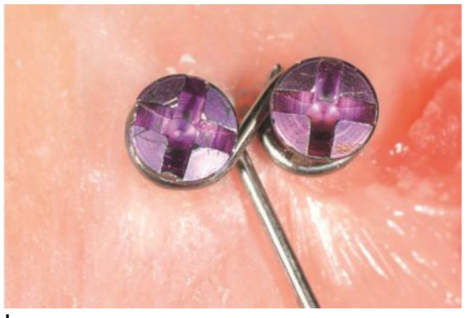

b

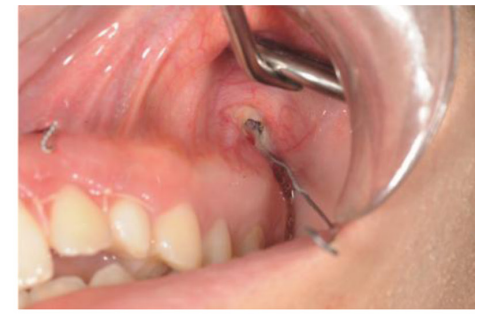

c

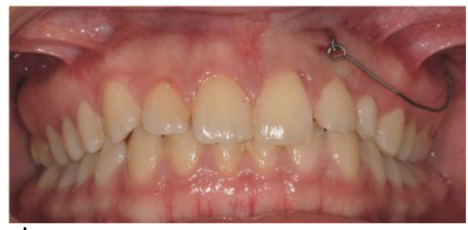

d
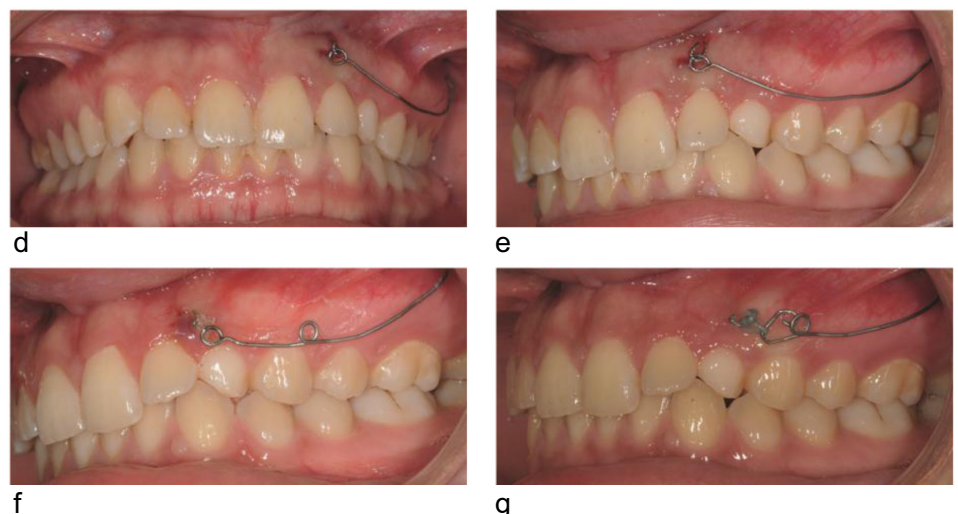

g

Figure 3

a) The position of the connector at rest points in the occlusovestibular direction due to the force. b) Direction of the loops, the spontaneous deactivation of the loop increases the disto-occlusal traction. c) Point of emergence in the vestibule.

$d$ and e) Insertion of the connector in the solid strand of 23. $f$ and $g$ ) Reduction of the length of the connection, the direction of the loop increases the occlusal traction.

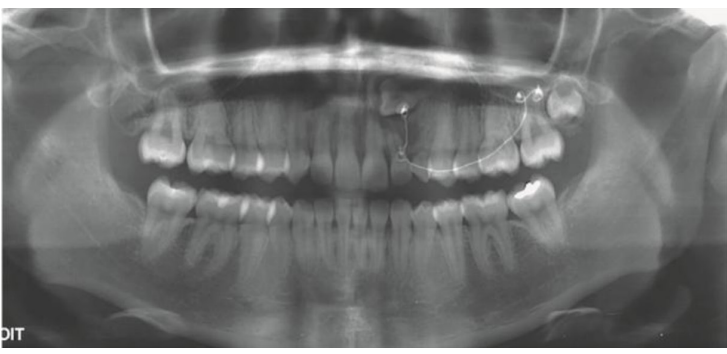

a

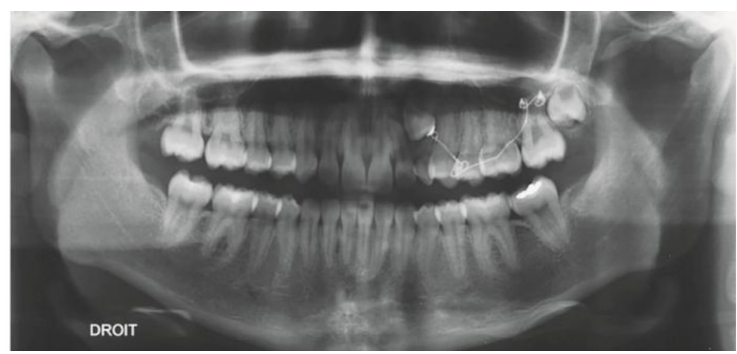

b

Figure $4 a, b$

Tracking path of 23. After avoiding 22, an occlusodistal path is created.

eyelet is attached to the cosmetic element to exert traction, which is gradually decreased to coincide with canine development (Figure 6).

Correction of the rotation of 23 by a rotational torque created by an auxiliary arm in the vestibule, followed by attaching an eyelet and finally by employing a "mirrored" fastener on 13 , which was designed at the beginning of the treatment (Fig. 6).

Step 5: An undesirable vestibular version of the maxillae and the ClassII incisors is observed after aligning the maxillary arch. Dual-top transmucosal 8-mm miniscrews are used for 


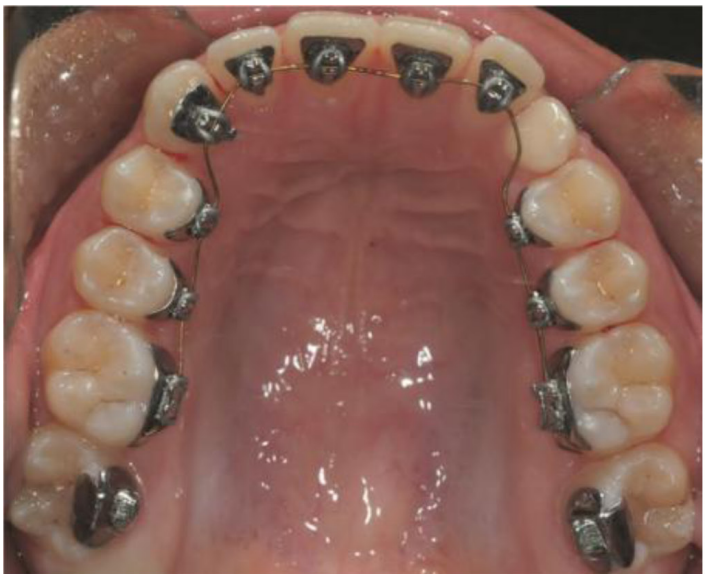

a

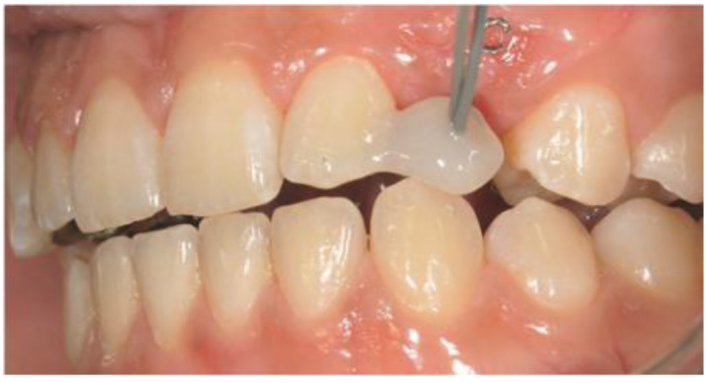

C

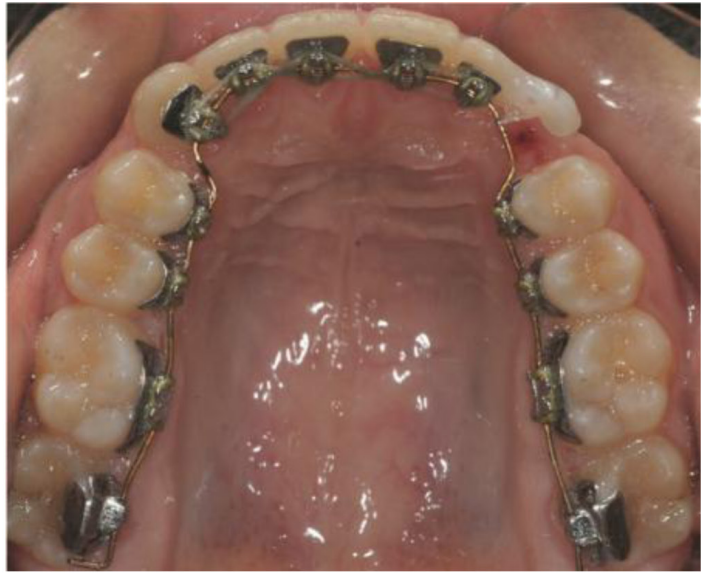

b

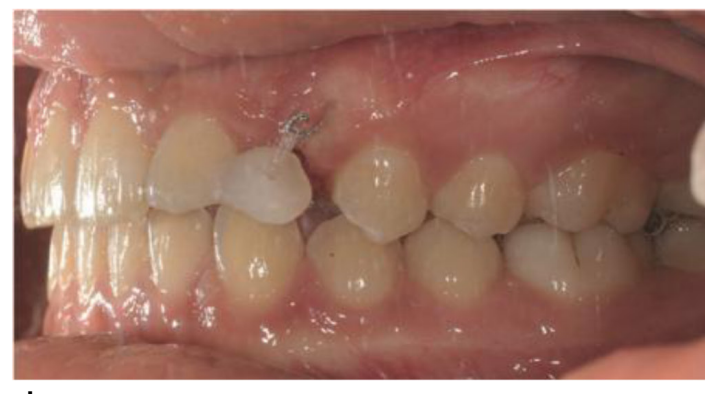

d

Figure $5 a, b, c, d$

Bonding the multi-fastener device, aligning, and leveling 22, avulsion 63, pulling 23 through a pierced hole in the cosmetic element.

the Class-II correction and closure of the maxillary spaces because the mandibular arch is not fitted (Fig. 7).

Step 6: Monitoring the static occlusion, canine slopes, and canine protection. As soon as a moderate, symmetrical, and bilateral occlusion is obtained with lateral movements, the device is removed, and a bonded compression wire is fitted (Figs. 8 and 9).

\section{CONCLUSION}

The combination of two major innovations in orthodontics-skeletal anchorage and the lingual technique with individualized fasteners-made it possible to perform a complex treatment with only a small number of braces for this young woman concerned about esthetics. The buried miniscrews and CT8 connector described by Chillès proved superior compared to other skeletal anchors for the placement of canines included in the vestibular 

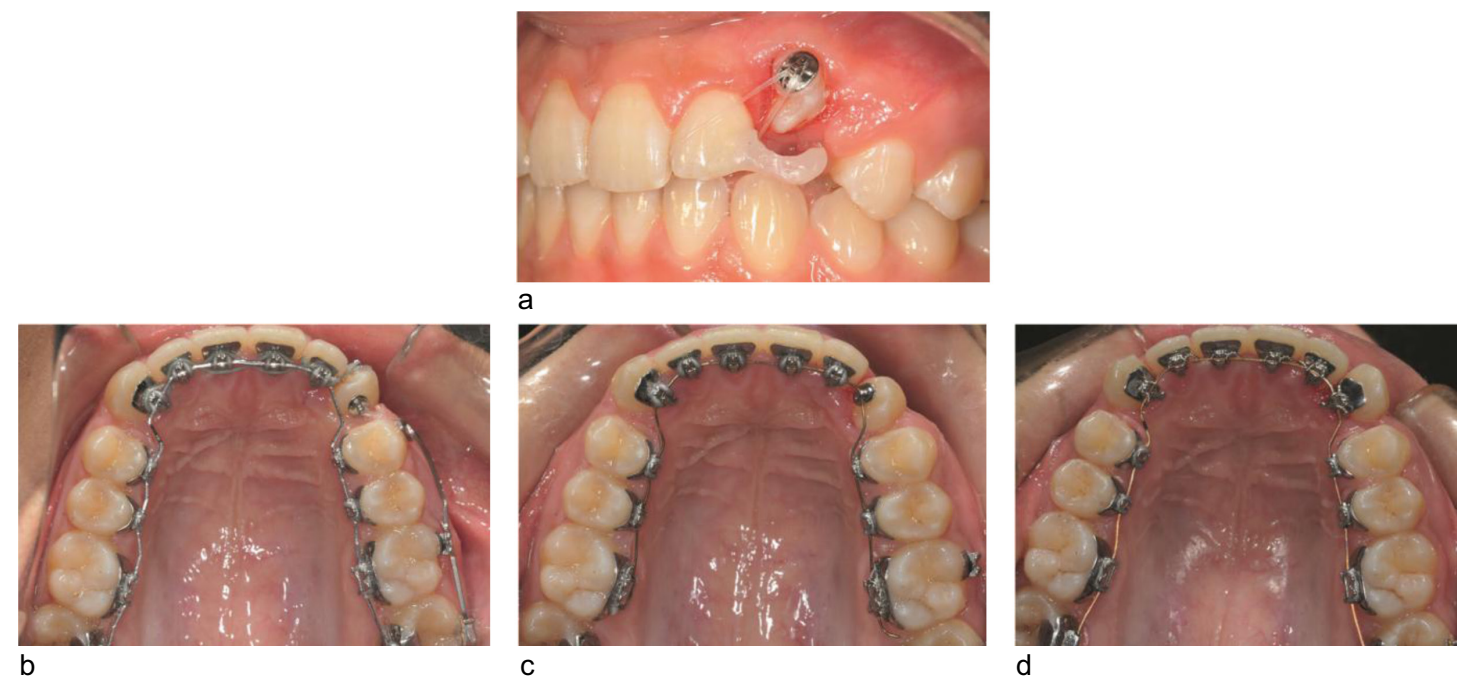

Figure $6 a, b, c, d$

Correction of the rotation of 23 .

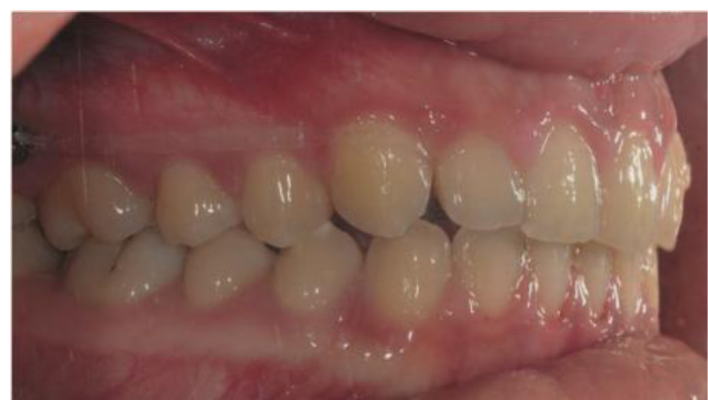

a

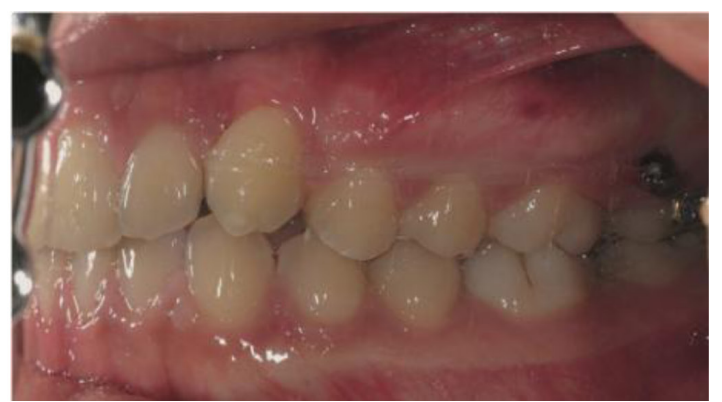

b

Figure $7 a, b$

Correction of class II, induced by alignment, by anchoring transmucosal miniscrews between the first and second molars.

sense, because of the length of the active arm and the direction of vestibular traction. The need to shape connectors in the mouth intraoperatively (bayonet for emergence in the middle of the vestibule and terminal coil) makes orthodontist consultation preferable while managing such patients, and enables orthodontists to place the device themselves. This is undoubtedly a psychological barrier to the use of this technique which nevertheless renders the best services. Orthodontists must be able to perform with adequate training this relatively minor procedure without difficulty and or be able to teach the surgeon the basics bending the connectors.

Conflict of interest: The author declares that there is no conflict of interest. 


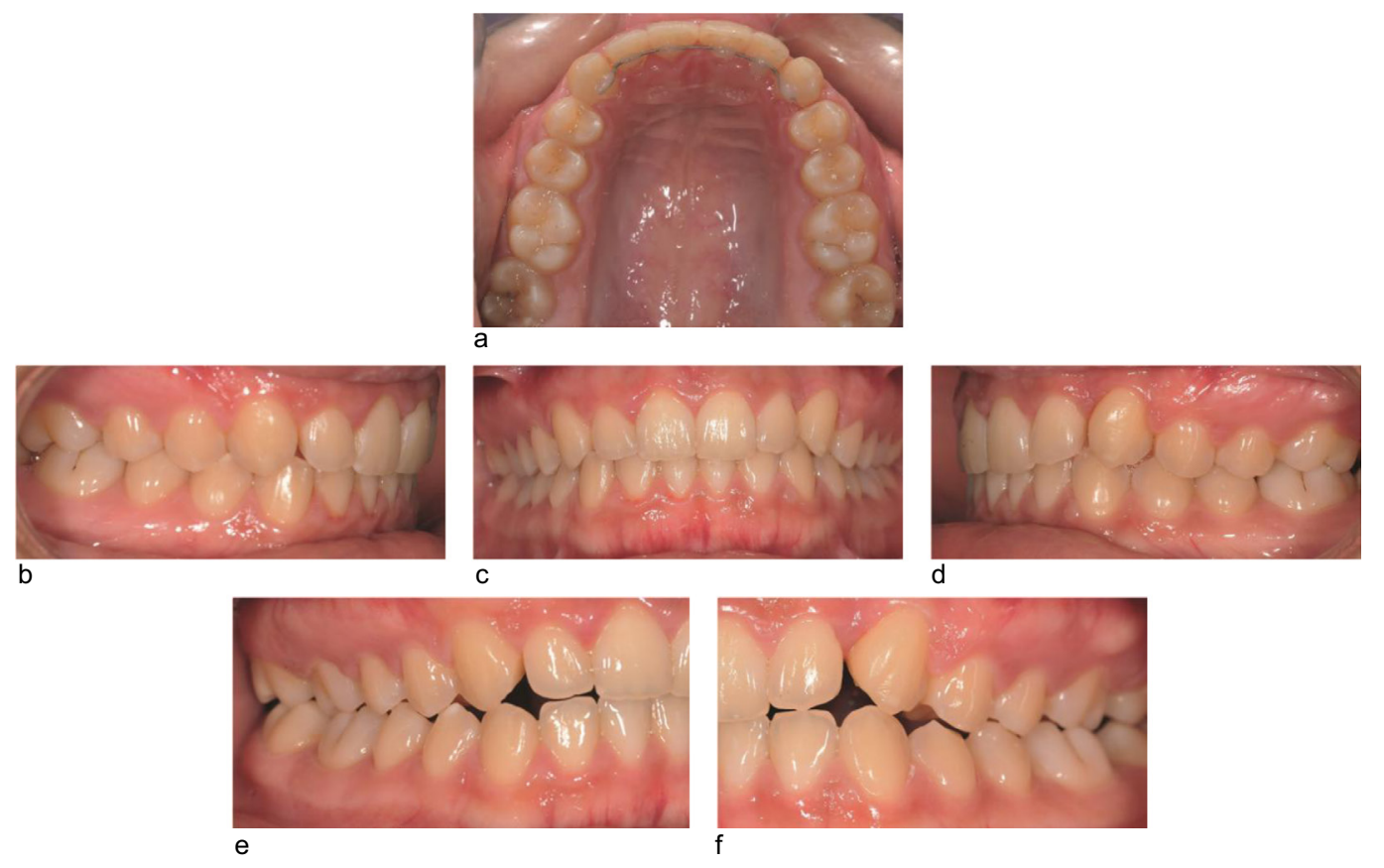

Figure $8 a, b, c, d, e, f$

End of treatment. A moderate and symmetrical disocclusion is sought after with lateral movements.

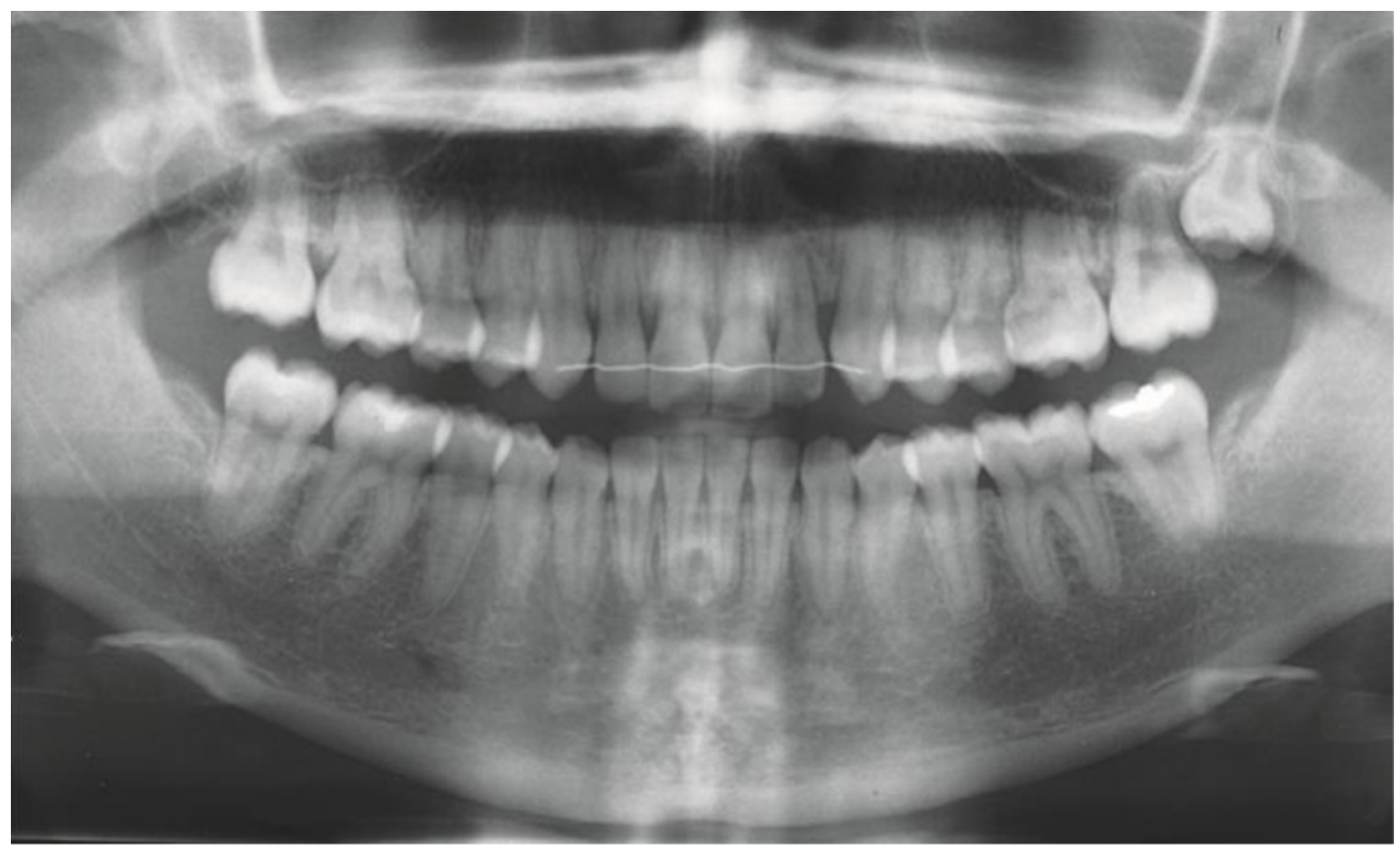

Figure 9

Panoramic view at the end of treatment: the germectomy of 28 is scheduled. 


\section{BIBLIOGRAPHY}

1. Brézulier D, Sorel $O$. The miniscrews: improvement of the outfit, new perspectives. Rev Orthop Dento Faciale 2016;50:265-274.

2. $D, J-G$ Chillès Chillès. A device to anchor skeletal traction and the implementation of the canines included. Rev Orthop Dento Faciale 2009;43(1):67-88.

3. Fleury JE, Deboets D, Assaad-Auclair C, Maffre N, Sultan P. The impacted canine. Review of 212 cases. General principles of treatment. Rev Stomatol Chir Maxillofac 1985;86(2):122-131.

4. Dersot JM. Periodontal surgery of the maxillary canine included in the service of the orthodontist. Proposal of a surgical decision tree. International Orthodontics. June 2017; 15(2):221-237.

5. Haim F. Association of dysmorphoses: canine inclusions, transposition and biproalveolina: application of miniscrews. Bioprogressive Orthod 2012;20:43-48.

6. Kyung HM, Park HS, Bae SM, Sung JH, Kim IB. Development of orthodontic mico-implants for intraoral anchorage. J Orthod 2003 blink;37:321-328.

7. McScherry PF. The ectopic maxillary canine: a review. Br J Orthod 1998;25(3):209-216.

8. French Society of Stomatology, Maxillofacial Surgery and Oral Surgery. Support for a canine: recommendation of good practice; July 2015.

9. Wiechmann D. New bracket system for lingual orthodontic treatment. Part 1: Theoretical background and development. Orofac Orthop / Fortschr Kieferorthop 2002;63:234-245. 